\title{
Design of Hydrophobic Nanoparticles for Spontaneous Translocation through Lipid Membranes
}

\author{
Yachong Guo', Marco Werner', Sergey Pogodin'1, Emmanuel Terazzi³, Ralf Seeman², Jean-Baptiste \\ Fleury $^{2}$, Vladimir Baulin ${ }^{1}$ \\ ${ }^{1}$ Departament d'Enginyeria Quimica, Universitat Rovira i Virgili \\ 26 Av. dels Paisos Catalans, 43007 Tarragona, Spain \\ Vladimir.baulin@urv.cat \\ ${ }^{2}$ Department of Inorganic and Analytical Chemistry, University of Geneva \\ 30 quai E. Ansermet, CH-1211 Geneva 4, Switzerland \\ ${ }^{3}$ Universitat des Saarlandes, Experimental Physics \\ 66123 Saarbruecken, Germany
}

Lipid bilayers represent a serious protective barrier for living cells protecting the interior from the outside environment. Design of nanomaterials able to cross lipid bilayers is a challenging task in nanotechnology. Large variety of shapes, sizes and surface coatings are used for the design of nanomaterials to overcome this barrier. However the potential barrier is quite high for carbon nanotubes [1] and nanoparticles [2] to cross the lipid bilayer to translocate by thermal motion. It is generally accepted that small hydrophobic nanoparticles are blocked by lipid bilayers and accumulate in the bilayer core, while nanoparticles with sizes larger than $5 \mathrm{~nm}$ can only penetrate cells through a slow energy-dependent processes such as endocytosis, lasting minutes.

In one example we show how variation of hydrophobicity of the nanoparticles can lead to passive translocation of nanoparticles through lipid bilayer [3]. This adsorption transition through reversible destabilization of the structure of the bilayer induces enhanced permeability for water and small solutes.

In another example, we demonstrate [4] that lipid-covered hydrophobic nanoparticles may translocate through lipid membranes by direct penetration within milliseconds. We identified the threshold size for translocation: nanoparticles with diameters smaller than $5 \mathrm{~nm}$ stay trapped in the bilayer, while nanoparticles larger than $5 \mathrm{~nm}$ insert into bilayer, open transient pore in the bilayer. Using the Single Chain Mean Field (SCMF) theory [5] a mechanism of passive translocation through lipid bilayers is proposed. Observing individual translocation events of gold nanoparticles with 1-dodecanethiol chains through DMPC bilayers we confirm the particle translocation and characterize the kinetic pathway in agreement with our numerical predictions. Mechanism relies on spontaneous pore formation in the lipid bilayer. The observed universal interaction behaviour of neutral and chemically inert nanoparticles with bilayer can be classified according to size and surface properties.

The translocation pathway numerically predicted by the SCMF theory is confirmed by studying single translocation events with time resolved optical fluorescence and electrophysiological measurements in our microfluidic setup. After the insertion of a hydrophobic nanoparticle into a bilayer, the lipid molecules reorganize and flip their tails towards the nanoparticle completely wrapping the nanoparticle. Such a lipid wrapped nanoparticle is only weakly bound to the lipid bilayer and forms a spontaneous pore that can open with thermal energies enabling passive translocation of nanoparticles. The measured lifetime of such a spontaneous pore during translocation was found to be on the order of few milliseconds and closes again without rupturing the bilayer. During the insertion process of a lipid coated nanoparticle into the bilayer the nanoparticle exchange its lipid coating with the bilayer and in turn extracts lipid molecules from the bilayer during a translocation event. However, as predicted by the theoretical analysis, the coating of a nanoparticle entering the bilayer is not the same when leaving the bilayer.

The reported lipid exchange between nanoparticles and lipid bilayer, which can be used to alter cell membrane composition, deliver "to" and "from" lipid bilayer. This mechanism provides numerous opportunities for biotechnological applications ranging from targeted biomaterial elimination and/or precise delivery to the membrane or trapping in the cells. 
Specific coating of nanoparticles opens perspectives to deliver objects as RNA, ligands or short biopolymers through the membrane or into the membrane.

\section{References}

[1] S. Pogodin and V. A. Baulin, "Can a Carbon Nanotube Pierce through a Phospholipid Bilayer?," ACS Nano, vol. 4, pp. 5293-5300, 2010.

[2] S. Pogodin and V. A. Baulin, "Equilibrium Insertion of Nanoscale Objects into Phospholipid Bilayers," Curr. Nanosci., vol. 7, no. 5, pp. 721-726, 2011.

[3] S. Pogodin, M. Werner, J.-U. Sommer, and V. A. Baulin, "Nanoparticle-Induced Permeability of Lipid Membranes," ACS Nano, p. 121112084603005, 2012.

[4] Y. Guo, E. Terazzi, R. Seemann, J. B. Fleury, and V. A. Baulin, "Direct proof of spontaneous translocation of lipidcovered hydrophobic nanoparticles through a phospholipid bilayer," Sci. Adv., vol. 2, no. 11, p. e1600261, 2016.

[5] S. Pogodin and V. A. Baulin, "Coarse-Grained Models of Phospholipid Membranes within the Single Chain Mean Field Theory,” Soft Matter, vol. 6, pp. 2216-2226, 2010. 